\title{
Frailty and the role of obliterative versus reconstructive surgery for pelvic organ prolapse; a national study
}

\author{
Anne M Suskind, MD, MS ${ }^{1}$, Chengshi Jin'ㄹ, Louise C. Walter, MD $^{3,4}$, and Emily Finlayson, \\ MD, MS ${ }^{5}$ \\ ${ }^{1}$ Department of Urology, University of California, San Francisco \\ ${ }^{2}$ Department of Epidemiology and Biostatistics, University of California, San Francisco \\ ${ }^{3}$ Division of Geriatrics, Department of Medicine, University of California, San Francisco \\ ${ }^{4}$ Division of Geriatrics, Veterans Affairs Medical Center, San Francisco, California \\ ${ }^{5}$ Department of Surgery, University of California, San Francisco
}

\section{Abstract}

Purpose-To determine whether frailty affects the type of pelvic organ prolapse (POP) surgery performed and the odds of postoperative complications.

\begin{abstract}
Materials and Methods-This is a retrospective cohort study of women undergoing obliterative and reconstructive surgery for POP in the American College of Surgeons National Surgical Quality Improvement Program (ACS NSQIP) from 2005 to 2013. We quantified frailty using the NSQIP Frailty Index (NSQIP-FI) and used logistic regression models predicting type of procedure (colpocleisis) and odds of post-operative complications.
\end{abstract}

Results-We identified a total of 12,731 women undergoing POP repairs, 5.3\% of which were colpocleisis procedures, from 2005-2013. Among women undergoing colpocleisis, the average age was 79.2 years and $28.5 \%$ had a NSQIP-FI of 0.18 or higher, indicating frailty. Women undergoing colpocleisis procedures had higher odds of being frail (OR 1.9 95\% CI 1.4-2.6 for NSQIP-FI 0.18 compared to NSQIP-FI 0) and were older aged (OR 486.4 95\% CI 274.6-861.7 for age $85+$ compared to $<65$ ). For all types of POP procedures, frailty increased the odds of complications (OR 2.8 95\% CI 1.8-3.0 for NSQIP-FI 0.18 compared to NSQIP-FI 0), after adjusting for age and type of POP procedure.

Conclusions-For POP surgery, age is more strongly associated with type of procedure performed than frailty, however, frailty is more strongly associated with postoperative complications than age. Furthermore, incorporating frailty into preoperative decision-making is important for improving expectations and outcomes among older women considering POP surgery.

Corresponding Author: Anne M Suskind, MD, MS, 400 Parnassus Ave, Box 0738, San Francisco, CA 94143, 415-476-1611, Fax: 415-476-8849, Anne.Suskind@ucsf.edu. 


\section{Introduction}

Pelvic organ prolapse is a major public health issue in the United States, particularly among older women. By the year 2050, it is estimated that there will be a $46 \%$ increase in affected women, ${ }^{1}$ many of whom will seek surgical treatment for this problem. The surgical treatment of older women, however, is not always as straightforward as in younger women, where important age-related considerations such as frailty may come into play. Frailty, defined as a decrease in physiologic capacity that predisposes individuals to poor outcomes in the face of stressors such as surgery, ${ }^{2-6}$ is recommended by the American College of Surgeons National Surgical Quality Improvement Program (ACS NSQIP) and the American Geriatrics Society (AGS) as part of the preoperative assessment in older adults. ${ }^{7}$

There are several surgical approaches of varying complexity for apical pelvic organ prolapse surgery. These approaches range from obliterative procedures, such as colpocleisis, to reconstructive procedures including abdominal, vaginal, and laparoscopic or robotic colpopexy. The advantages of the obliterative approach include the option for local anesthesia, shorter operative times, lower blood loss and faster recovery. ${ }^{8}$ These benefits, in combination with extremely high success rates upwards of $90 \%,{ }^{9-12}$ makes this procedure ideally suited for frail older individuals who are not sexually active. The real-world use and outcomes of this obliterative procedure in comparison to the other reconstructive procedures, however, has yet to be explored.

Using data from the ACS NSQIP, from 2005 to 2013, we examined the following three objectives: (1) to define the demographic and case-based characteristics of women who underwent obliterative versus reconstructive surgery for pelvic organ prolapse, (2) to define the role of frailty as a determinant of the obliterative approach, and (3) to identify whether frailty associated with complications for all types of pelvic organ prolapse repairs during the study period.

\section{Methods}

\section{Patients and Databases}

This is a retrospective cohort study of women who underwent apical pelvic organ prolapse procedures between 2005 and 2013 in the ACS NSQIP Participant Data Use File. This study was deemed exempt by our institution's Institutional Review Board (IRB). The ACS NSQIP database is a validated national multicenter database that is prospectively maintained by certified surgical reviewers at each site. Reviewers extract data from patient records and obtain postoperative clinical data and outcomes up to 30 days after the date of the procedure. ${ }^{13}$ Interrater disagreement across all variables in the ACS NSQIP dataset is extremely low at $1.5 \%$, with kappa values suggesting substantial or almost perfect agreement for most variables. ${ }^{14}$

We identified pelvic organ prolapse cases by Common Procedural Terminology (CPT) codes for each procedure: 57120 [colpocleisis (Le Fort type)]; 57280 (colpopexy, abdominal approach); 57282 (colpopexy vaginal approach) and 57283 [colpopexy, intra-peritoneal 
approach (uterosacral levator myorrhaphy)]; 57425 [laparoscopy, surgical, colpopexy (suspension of vaginal apex)].

\section{Outcomes}

We had two primary outcomes in this study. The first outcome was choice of procedure being a colpocleisis and the second outcome was risk of complications. In order to determine complications, we created a composite variable for all of the following 30-day complications: superficial wound infection, deep wound infection, pneumonia, blood transfusion, renal failure, deep vein thrombosis (DVT), myocardial infarction (MI), dehiscence, stroke, pulmonary embolus (PE), coma, cardiac arrest, sepsis, septic shock, reintubation, return to the operating room, discharge to a skilled or assisted living facility (variable added in 2011) and mortality.

\section{Covariates}

We used the NSQIP Frailty Index (NSQIP-FI) to describe frailty in our cohort. The NSQIPFI is a frailty index that was adapted from the Canadian Study of Health and Aging-Frailty Index for specific use in the ACS NSQIP data. It is a cumulative deficits model that includes the following criteria: history of diabetes, impaired functional status, history of chronic obstructive pulmonary disease or pneumonia, history of congestive heart failure, history of MI within 6 months of surgery, history of percutaneous coronary intervention, cardiac surgery or angina, being on hypertensive medications, peripheral vascular disease or rest pain, impaired sensorium, transient ischemic attack or cerebral vascular accident defined as focal neurologic deficits of sudden onset and brief duration, and cerebrovascular accident with deficit defined by history of cerebrovascular accident with persistent residual dysfunction. The NSQIP-FI score is the sum of the variables present in an individual divided by the total number of variables (11) and is reported as 0.09 for $1 / 11$ deficits, 0.18 for 2 out of 11 deficits, and so on. ${ }^{15}$

Additional covariates include the calendar year of the operation, age, race, body mass index (BMI), smoking status and recent weight loss, surgical setting (inpatient versus outpatient), type of anesthesia [general, monitored anesthesia care (MAC), or other], and operative time (per hour). Additionally, in order to adjust for concurrent procedures performed at the time of pelvic organ prolapse repair, we examined the presence of procedures for stress urinary incontinence (SUI), hysterectomy and anterior and/or posterior pelvic organ prolapse repairs. Supplementary table 1 shows the CPT codes used for each procedure type.

\section{Statistical Analysis}

For our first study objective, to define the demographic and case-based characteristics of women who underwent obliterative versus reconstructive surgery for pelvic organ prolapse, variables were compared between colpocleisis procedures and reconstructive procedures for pelvic organ prolapse (e.g., abdominal colpopexy, vaginal colpopexy, and laparoscopic/ robotic colpopexy) using Chi-square tests and Mann-Whitney tests for significance where appropriate. 
The second objective of the study was to define the determinants of the obliterative approach (i.e., frailty), representing a case control methodology. Herein, logistic regression was performed to determine procedure type (colpocleisis). Finally, the third objective, to identify predictors (e.g., frailty) of complications associated with all types of pelvic organ prolapse repairs also employed case control methodology and logistic regression to determine 30-day post operative complications as a composite outcome. Both models were adjusted for variables that might impact the surgical decision-making process including calendar year, age, race, BMI, smoking status, recent weight loss and NSQIP-FI. The model looking at risk of complications was adjusted for all variables included in the previous model, in addition to the type of pelvic organ prolapse procedure, surgical setting, type of anesthesia, operative time, number of days from operation to discharge, and the presence of concurrent procedures for SUI, hysterectomy, and anterior and/or posterior pelvic organ prolapse repair. All analyses were performed sing SAS 9.4.

\section{Results}

\section{Characteristics of women who underwent obliterative versus reconstructive surgery}

There were 12,731 procedures for apical pelvic organ prolapse, $676(5.3 \%)$ of which were colpocleisis procedures, from 2005 to 2013 in the ACS NSQIP database (Table 1).

Compared to the various types of reconstructive procedures (abdominal colpopexy, vaginal colpopexy and laparoscopic/robotic colpopexy), women who underwent colpocleisis procedures tended to be older (52.4\% were ages $75-84$ in the obliterative group compared to $11.0 \%$ in the reconstructive group and $25.4 \%$ were ages 85 and older in the obliterative group compared to $1.1 \%$ in the reconstructive group, p's<0.001) and more frail $(22.9 \%$ NSQIP-FI 0.18 in the obliterative group compared to $9.3 \%$ in the reconstructive group and $5.6 \%$ NSQIP-FI $0.27+$ in the obliterative group compared to $1.2 \%$ in the reconstructive group, p's $<0.001$ ) compared to their reconstructive counterparts. Additionally, individuals undergoing colpocleisis had a lower frequency of being white (87.7\% compared to $92.7 \%$, $\mathrm{p}<0.001$ ), had lower BMIs (average 26.6 compared to 28.1, $\mathrm{p}<0.001$ ), were less likely to be smokers (3.3\% compared to $10.0 \%, \mathrm{p}<0.001$ ), had a higher frequency of having types anesthesia other than general or MAC (14.5\% compared to 3.4\%, $<<0,001)$, had shorter operative times (mean 1.92 hours compared to 2.66 hours, $\mathrm{p}<0.001$ ) and had fewer concurrent procedures for stress urinary incontinence (35.5\% compared to $37.5 \%, \mathrm{p}<0.001$ ), hysterectomy $(13.3 \%$ compared to $59.0 \%, \mathrm{p}<0.001)$ and anterior and/or posterior organ prolapse repairs $(18.0 \%$ compared to $44.5 \%, \mathrm{p}<0.001)$.

\section{Determinants of an obliterative approach}

The logistic regression model predicting colpocleisis as the type of pelvic organ prolapse procedure performed is shown in Table 2. Increasing NSQIP-FI was associated with increasing odds of having a colpocleisis (OR 1.3 95\% CI 1.0-1.7 for NSQIP-FI 0.09; OR 1.9 95\% CI 1.4-2.6 for NSQIP-FI 0.18; OR 2.2 95\% CI 1.3-3.7 for NSQIP-FI 0.27+ all compared to NSQIP-FI 0). Additionally, increasing age (OR 13.3 95\% CI 7.8-22.8 for ages 65-74; OR 91.3 95\% CI 54.0-154.5 for ages 75-84; OR 486.4 95\% CI 274.6-861.7 for ages $85+$ all compared to ages < 65), races other than white or black (OR $3.495 \%$ CI 2.3-5.1 
compared to white), and being underweight (OR 2.3 95\% CI 1.3-4.3 compared to normal weight) were associated with an increased odds of having a colpocleisis procedure.

\section{Predictors of postoperative complications}

The logistic regression model predicting surgical complications is shown in Table 3 . Increasing NSQIP-FI is associated with increased odds of complications (OR 1.4, 95\% CI 1.1-1.7; OR 2.3 95\% CI 1.8-3.0 for NSQIP-FI 0.18; OR 12.1 95\% CI 8.4-17.6 for NSQIPFI 0.27+ compared to NSQIP-FI of 0). Type of pelvic organ prolapse procedure, either colpocleisis, abdominal colpopexy, vaginal colpopexy or laparoscopic/robotic colpopexy was not associated with statistically increased or decreased odds of post-operative complications. Other statistically significant predictors of complications included age greater than 85 (OR 2.0 95\% CI 1.3-3.2), smoking (OR 1.4 95\% CI 1.1 -1.8) and longer operative time (OR 1.2 95\% CI 1.1-1.3 per hour), while having an outpatient procedure compared to an inpatient procedure was associated with lower odds of postoperative complications (OR $0.795 \%$ CI 0.6-0.8).

\section{Discussion}

We found that age, to a greater extent than frailty, was strongly associated with having a colpocleisis procedure (as opposed to a reconstructive procedure) and that frailty, to a greater extent than age, was associated with increased odds of post-operative complications among women undergoing all types of pelvic organ prolapse procedures. Taken together, our findings indicate that surgeons tend to select women for colpocleisis procedures based on age, but that it is frailty that has a stronger association with postoperative complications.

Prior studies found that $16 \%$ of women undergoing pelvic organ prolapse surgery were categorized as "frail" using the Fried criteria and $30.7 \%$ reported functional difficulty or dependence in performing at least $1 \mathrm{ADL} .{ }^{16}$ Our study found that rates frailty differed based on type of apical prolapse procedure performed, whereby that $28.5 \%$ of women undergoing colpocleisis procedures had a NSQIP-FI $\searrow 0.18$, and only $10.1 \%, 11.7 \%$, and $8.1 \%$ of women undergoing abdominal colpopexy, vaginal colpopexy, and laparoscopic/robotic colpopexy were frail, respectively.

Another study using ACS NSQIP data showed that mortality and complications following colpocleisis procedures are low, with the most common reported complication being urinary tract infections at 5.8\%. ${ }^{8}$ In another study, objective and subjective cure rates following colpocleisis in women over the age of 80 have been shown to be similar to those among younger women, ${ }^{17}$ further promoting their safety and benefit in the older female population.

We found it somewhat surprising that neither age (with the exclusion of age $>85$ ) nor the type of prolapse procedure (either obliterative or reconstructive) influenced the odds of postoperative complications. This finding suggests that patient characteristics, such as frailty, are more important predictors of adverse outcomes than the type of procedure itself.

Our findings should be considered with certain limitations in mind. First, we do not have access to women who did not undergo surgery and were perhaps treated more conservatively 
with pelvic floor physical therapy, a pessary, or no treatment at all. This may lead to positive selection bias, wherein the women chosen to have surgery were healthier than the women selected not to have surgery. Despite this limitation, however, we did find a statistically significant association between frailty and both outcomes of interest (having a colpocleisis and post-operative complications), suggesting that we may in fact be underestimating the deleterious effects of frailty on these outcomes. Second, we are somewhat limited by the nature of this registry database in the level of detail that we have available for our analyses. For example, obliterative procedures are only a viable option among women who are not sexually active, since they "obliterate" the vaginal space, however, we do not have access to data on sexual activity via this database. While it would be nice to have more granular data on variables pertaining to sexual activity, severity of prolapse, patient preferences and other clinical items, the strength of these data lie in the fact that they are taken from a national sample of hospitals that participate in the ACS NSQIP database, adding power and strength to the study findings beyond information that a single-center study can provide. Third, we used a composite outcome for complications, which included a wide range of adverse events from superficial wound infection to cardiac arrest and coma. All complications are weighted equally in this outcome, yet not all complications are similar in severity, which should be considered when interpreting the results of the study.

\section{Conclusions}

Our study found that age, to a greater extent than frailty, was associated with having an obliterative, rather than a reconstructive, surgery for pelvic organ prolapse repair but that frailty was more strongly associated with increased post-operative complications. These findings indicate that surgeons rely heavily on age for procedure selection, but hat frailty may be a more informative indicator for this purpose and should be incorporated into the perioperative decision-making process.

\section{References}

1. Wu JM, Hundley AF, Fulton RG, Myers ER. Forecasting the prevalence of pelvic floor disorders in U.S. Women: 2010 to 2050. Obstetrics and gynecology. 2009; 114(6):1278-1283. [PubMed: 19935030]

2. Fried LP, Tangen CM, Walston J, et al. Frailty in older adults: evidence for a phenotype. The journals of gerontology Series A, Biological sciences and medical sciences. 2001; 56(3):M146-156.

3. Suskind AM, Walter LC, Jin C, et al. Impact of frailty on complications in patients undergoing common urological procedures: a study from the American College of Surgeons National Surgical Quality Improvement database. BJU international. 2016; 117(5):836-842. [PubMed: 26691588]

4. Suskind AM, Jin C, Cooperberg MR, et al. Preoperative Frailty Is Associated With Discharge to Skilled or Assisted Living Facilities After Urologic Procedures of Varying Complexity. Urology. 2016

5. Robinson TN, Wu DS, Sauaia A, et al. Slower walking speed forecasts increased postoperative morbidity and 1-year mortality across surgical specialties. Annals of surgery. 2013; 258(4):582588. discussion 588-590. [PubMed: 23979272]

6. Robinson TN, Walston JD, Brummel NE, et al. Frailty for Surgeons: Review of a National Institute on Aging Conference on Frailty for Specialists. Journal of the American College of Surgeons. 2015; 221(6):1083-1092. [PubMed: 26422746]

7. Mohanty S, Rosenthal RA, Russell MM, Neuman MD, Ko CY, Esnaola NF. Optimal Perioperative Management of the Geriatric Patient: A Best Practices Guideline from the American College of 
Surgeons NSQIP and the American Geriatrics Society. Journal of the American College of Surgeons. 2016; 222(5):930-947. [PubMed: 27049783]

8. Catanzarite T, Rambachan A, Mueller MG, Pilecki MA, Kim JY, Kenton K. Risk factors for 30-day perioperative complications after Le Fort colpocleisis. The Journal of urology. 2014; 192(3):788792. [PubMed: 24641911]

9. Fitzgerald MP, Richter HE, Bradley CS, et al. Pelvic support, pelvic symptoms, and patient satisfaction after colpocleisis. International urogynecology journal and pelvic floor dysfunction. 2008; 19(12):1603-1609. [PubMed: 18690402]

10. DeLancey JO, Morley GW. Total colpocleisis for vaginal eversion. American journal of obstetrics and gynecology. 1997; 176(6):1228-1232. discussion 1232-1225. [PubMed: 9215178]

11. Harmanli OH, Dandolu V, Chatwani AJ, Grody MT. Total colpocleisis for severe pelvic organ prolapse. The Journal of reproductive medicine. 2003; 48(9):703-706. [PubMed: 14562635]

12. Goldman J, Ovadia J, Feldberg D. The Neugebauer-Le Fort operation: a review of 118 partial colpocleises. European journal of obstetrics, gynecology, and reproductive biology. 1981; 12(1): 31-35.

13. American College of Surgeons National Quality Improvement Program (ACS NSQIP). 2014 Semiannual Report.

14. Shiloach M, Frencher SK Jr, Steeger JE, et al. Toward robust information: data quality and interrater reliability in the American College of Surgeons National Surgical Quality Improvement Program. Journal of the American College of Surgeons. 2010; 210(1):6-16. [PubMed: 20123325]

15. Velanovich V, Antoine H, Swartz A, Peters D, Rubinfeld I. Accumulating deficits model of frailty and postoperative mortality and morbidity: its application to a national database. The Journal of surgical research. 2013; 183(1):104-110. [PubMed: 23415494]

16. Erekson EA, Fried TR, Martin DK, Rutherford TJ, Strohbehn K, Bynum JP. Frailty, cognitive impairment, and functional disability in older women with female pelvic floor dysfunction. International urogynecology journal. 2015; 26(6):823-830. [PubMed: 25516232]

17. Krissi H, Aviram A, Ram E, Eitan R, Wiznitzer A, Peled Y. Colpocleisis surgery in women over 80 years old with severe triple compartment pelvic organ prolapse. European journal of obstetrics, gynecology, and reproductive biology. 2015; 195:206-209. 


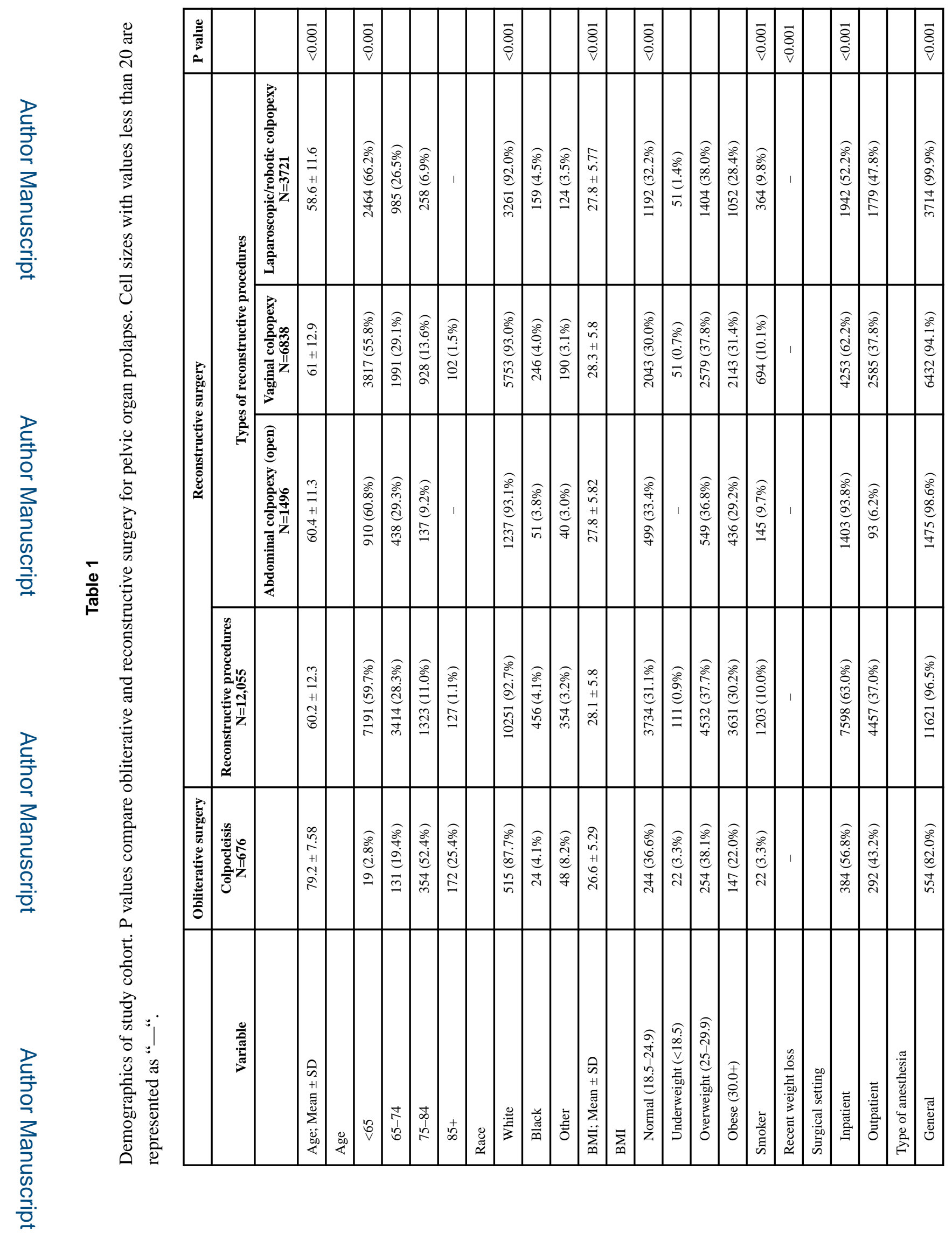

J Urol. Author manuscript; available in PMC 2017 June 15. 


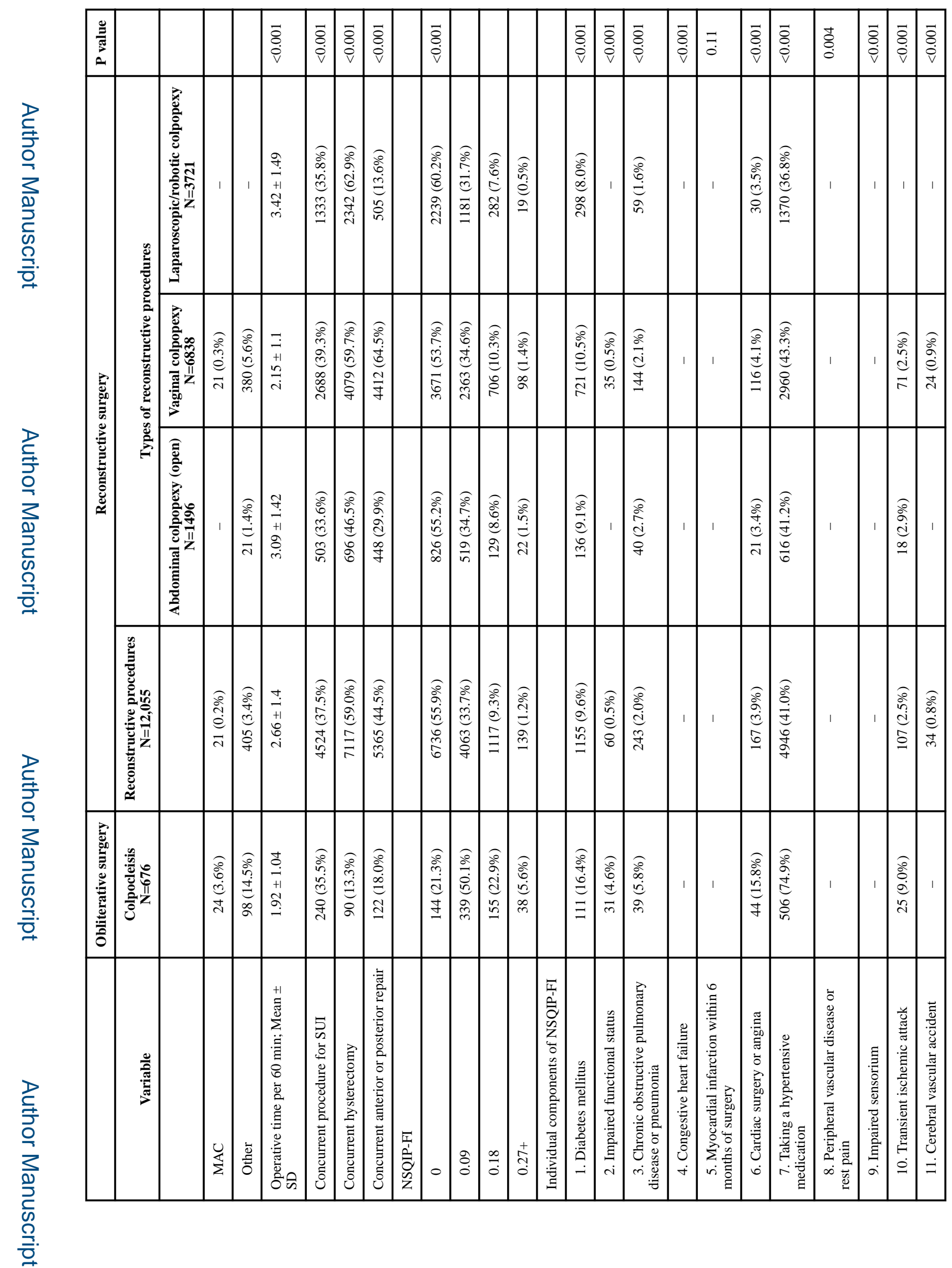

J Urol. Author manuscript; available in PMC 2017 June 15. 

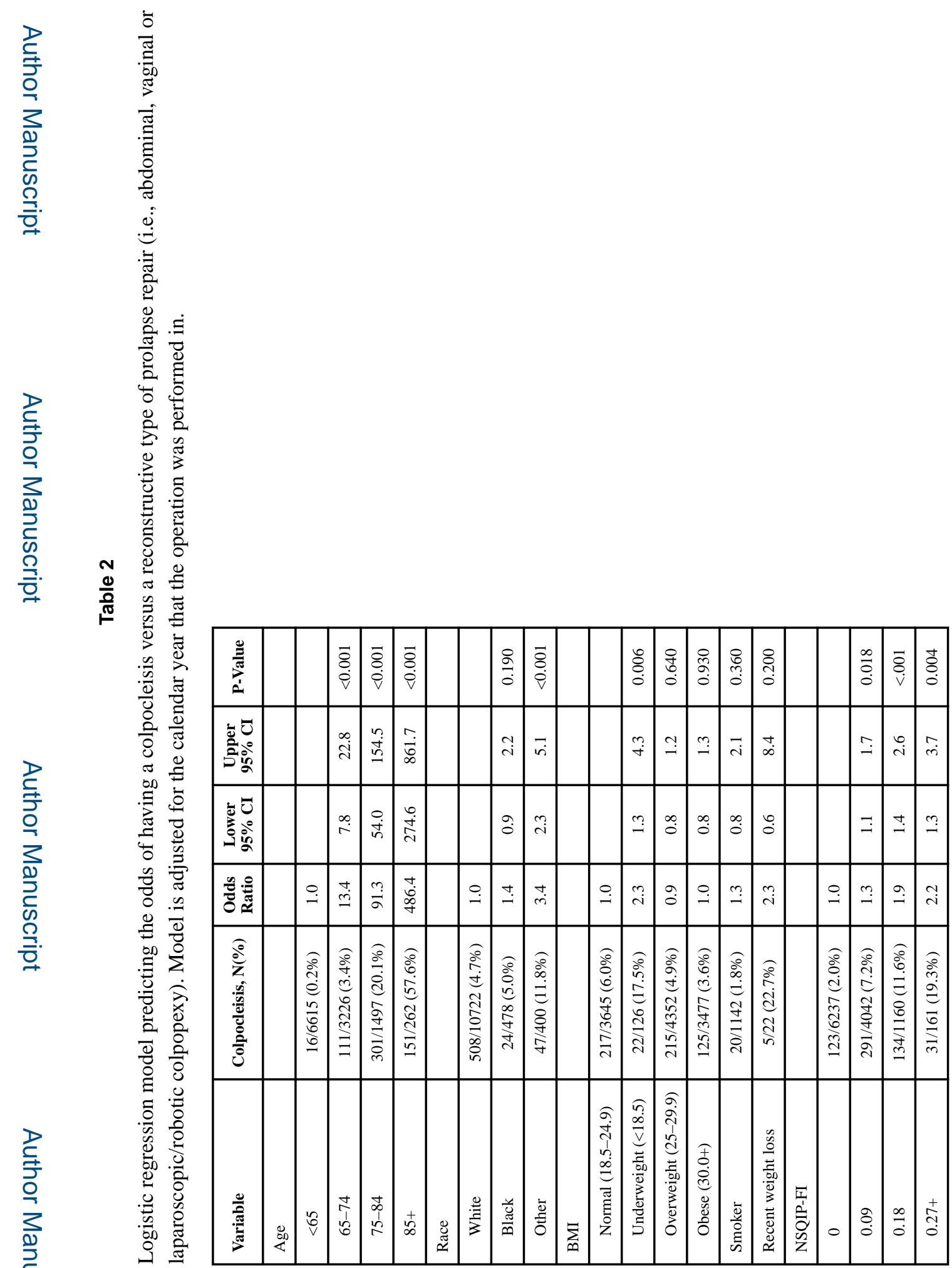

을

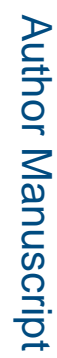




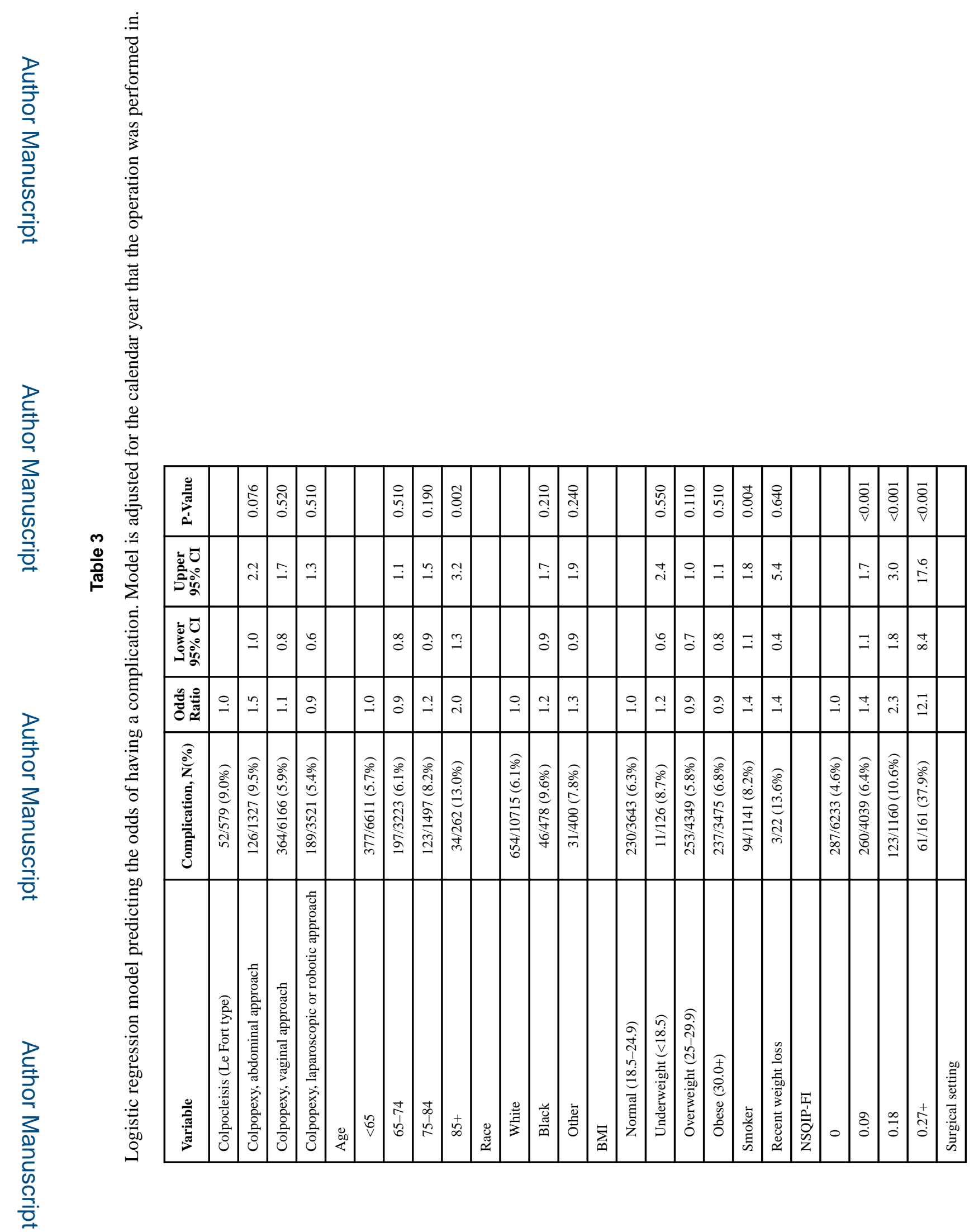




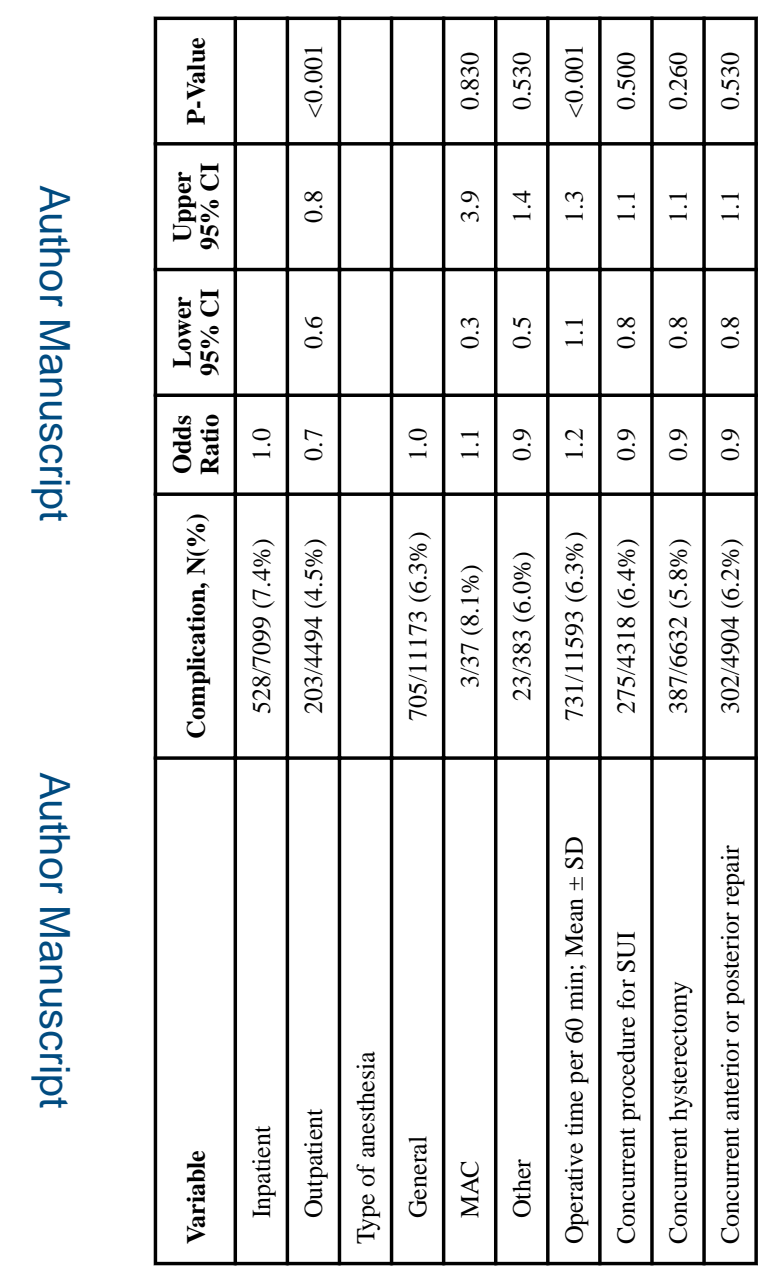

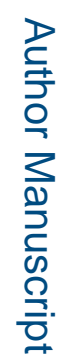

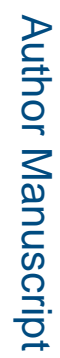

